
\title{
25 Research Soure \\ Flight of the COVID-19 patient: experience with aWuhan evacuee- a case report
}

\section{Sandeep Segar}

University of California San Diego

Daniel Bouland

University of California San Diego

Francesca Torriani

University of California San Diego

Kevin Kwak

University of California San Diego

Deepak Asudani

University of California San Diego

Randy Taplitz

University of California San Diego

Vineet Gupta（ $\sim$ v1gupta@health.ucsd.edu )

University of California San Diego https://orcid.org/0000-0003-4639-3316

\section{Case Report}

Keywords: 2019 novel coronavirus, SARS-CoV-2, COVID-19, severe acute respiratory syndrome coronavirus 2 , remdesivir, quarantine

Posted Date: April 23rd, 2020

DOI: https://doi.org/10.21203/rs.3.rs-24578/v1

License: (c) (i) This work is licensed under a Creative Commons Attribution 4.0 International License.

Read Full License 


\section{Abstract}

Background:Coronavirus disease 2019 (COVID-19), caused by severe acute respiratory syndrome coronavirus 2 (SARS-CoV-2), was declared a global pandemic by World Health Organization in March 2020.

Case Presentation:We report a case of a 51-year-old Chinese woman who was evacuated from Wuhan, China and diagnosed with COVID-19 infection at a Southern California quarantine facility. Clinical course was notable for high fevers, night sweats, productive cough, transient leukopenia, lymphopenia, thrombocytopenia, and transaminitis. Evolving hypoxia and infiltrates on chest imaging warranted the trial of an investigational antiviral drug - remdesivir. The patient recovered and was discharged after two weeks of hospitalization.

Conclusion:This case highlights the patient's clinical course including diagnostic work-up, medical management, and challenges in defining non-infectivity in a relatively unknown disease.

\section{Introduction}

A cluster of unexplained pneumonia cases linked to the Huanan seafood market in Wuhan, China was first reported on December 31, 2019.[1] After negative testing for common respiratory viruses, these patients tested positive for a novel coronavirus - severe acute respiratory syndrome-related coronavirus 2 (SARS-CoV-2), which is the cause of coronavirus disease 2019 (COVID-19).[2,3] Some of these initial patients demonstrated hypoxemia, ground glass opacification on chest imaging, abnormal laboratory results-low white blood cell counts (WBC), low absolute lymphocyte counts (ALC), low platelet counts, elevated liver enzymes, and elevated creatinine.[2]

COVID-19 was declared a global pandemic by the World Health Organization with over 750,000 confirmed cases in over two hundred countries and territories as of March 31,2020.[4] We report the case of an early COVID-19 patient who was evacuated from Wuhan, China and developed signs of infection at a Southern California quarantine facility. This case highlights the patient's clinical course including relevant history, diagnostic work-up, medical management, and challenges in defining non-infectivity.

\section{Case Presentation}

A 51-year-old Chinese woman with no significant past medical history presented to our institution with a one-day history of fevers, chills, sweats, nonproductive cough from a Southern California quarantine facility. She had worked as a nurse in an outpatient medicine clinic in Wuhan, China, where she cared for patients with upper respiratory symptoms but without confirmed diagnoses of COVID-19. While caring for these patients, she reported wearing a standard surgical mask and gloves. Three weeks prior to hospital admission, upon becoming aware of a rapidly spreading pulmonary infection within her community, the patient took leave from work and self-isolated herself in her apartment with her husband and grandson, neither of whom had signs of infection. She had no direct exposure to the Huanan seafood market. 
Four days prior to hospital admission, she and her grandson were evacuated on a flight from the Wuhan International Airport. Late the next day, she arrived at a Southern California government facility for an intended fourteen-day quarantine. One day prior to admission, the patient developed nonproductive cough, fevers, chills and sweats. The same day, nasopharyngeal (NP) and oropharyngeal (OP) swabs for COVID-19 using reverse transcription polymerase chain reaction (RT-PCR) were sent from the quarantine facility to the US Centers for Disease Control and Prevention (CDC) laboratory per recommended guidelines. * (see appendix)

The following day, she was admitted to our institution and was placed in contact, droplet, and airborne isolation precautions per CDC recommendations. At admission, her temperature was $38.4 \llbracket C$, blood pressure was 101/69 mm Hg, heart rate was 84 beats per minute, respiratory rate was 17 breaths per minute, and oxygen saturation was $96 \%$ on room air. Physical examination including cardiopulmonary evaluation was unremarkable. Laboratory studies were notable for WBC 3600 per $\mathrm{mm}^{3}$ (range 4100$10,400 / \mathrm{mm}^{3}$ ), absolute neutrophil count (ANC) 2100 per $\mathrm{mm}^{3}$, ALC 1000 per $\mathrm{mm}^{3}$, and platelet count 121,000 per $\mathrm{mm}^{3}$. Other tests including liver function tests, coagulation studies, procalcitonin, and urinalysis were unremarkable. A NP swab using RT-PCR was negative for usual viral pathogens, including influenza $A / B$, respiratory syncytial virus, human rhinovirus/enterovirus, human metapneumovirus, parainfluenza, and four common coronavirus strains previously known to cause human illness (229E, HKU1, NL63, and OC43). Chest x-ray demonstrated clear lung fields bilaterally without consolidation or effusion (Figure 2A). Serial NP and OP swabs were tested every other day per CDC guidance to evaluate clearance of infection. No antibiotics were given to the patient. NP and OP swabs from the day prior to admission returned positive for COVID-19, and the patient became the fourteenth confirmed case in the United States.

During days 1-6 of hospitalization, she experienced daily fevers, chills, drenching night sweats, and a nonproductive cough. By day 3 , she had dyspnea with minimal exertion, exacerbated by coughing fits. She also had diminished appetite with occasional nausea. She denied abdominal pain, diarrhea or dysuria. Between days 3 and 6, maximum temperature (Tmax) ranged from 39.0凶C to 39.7凶C (Figure 1). Blood pressure remained in low normal range. Pulmonary exam demonstrated bibasilar crackles without labored breathing. On hospital day 3 , her chest $x$-ray demonstrated new findings of bilateral lower lobe reticular opacities (Figure 2B). Platelets reached a nadir of 101,000 per $\mathrm{mm}^{3}$ on day 4, before improving. WBC improved to 5100 per $\mathrm{mm}^{3}$ on hospital day 5 , however, ALC decreased to 600 per $\mathrm{mm}^{3}$ on day 6 , before recovering (Figure 1). Liver enzymes remained normal until day 6 when aspartate aminotransferase (AST) rose to $50 \mathrm{U} / \mathrm{L}$ (Figure 3). Transthoracic echocardiogram was unremarkable. Chest $X$ ray on day 6 showed worsened bibasilar reticular opacification (Figure 2C). Blood cultures remained negative during hospitalization. TB Quantiferon resulted negative. Treatment was largely supportive during this time comprising of intravenous hydration and antipyretic therapy (acetaminophen $<4 \mathrm{~g}$ daily, ibuprofen, and axillary ice packs). 
On day 6 of hospitalization, due to persistent high fevers, relative hypoxemia, evolving chest x-ray findings, and mildly abnormal liver function, patient was initiated on compassionate use remdesivir 200 $\mathrm{mg}$ intravenous on day 1 followed by $100 \mathrm{mg}$ daily. Remdesivir, an investigational antiviral for COVID-19, was previously used in Ebola virus disease, severe acute respiratory syndrome coronavirus (SARS-CoV) and Middle East respiratory syndrome coronavirus (MERS-CoV). [5] Her fever curve improved on day 8 (Figure 1). The patient continued to have mild dyspnea, nausea, poor appetite, and fatigue. Her cough became productive of scant white sputum. Alanine aminotransferase (ALT) peaked at $84 \mathrm{U} / \mathrm{L}$ on day 10 , before trending down (Figure 3). As of hospital day 11, she remained afebrile off antipyretics. Oxygen saturations improved to $97 \%$ on ambient air. Her dyspnea and auscultatory rales resolved, however a mild productive cough persisted.

On hospital day 9, the CDC reported serial NP and OP swabs from her entire hospitalization to be negative (Figure 4). This included samples at the height of illness severity (days 1 to 7 ). Due to concerns with the adequacy of negative OP and NP testing in defining infection clearance, sputum PCR for COVID-19 on days 10 and 11 were obtained. Surprisingly, both sputum samples returned positive (Figure 4). Given the possibility that her productive cough harbored transmissible live virus, the patient was discharged on day 15 to a government medical facility for continued isolation until the resolution of all symptoms. At the time of transfer, she remained afebrile and asymptomatic apart from a mild productive cough. Patient returned home after 19 days at the government isolation facility after resolution of her cough. She remained non-symptomatic at home when last followed up.

\section{Discussion}

Our case helps offer insight into the clinical course of COVID-19 patients. Coronavirus disease is thought to be transmitted from person-to-person by respiratory droplets and direct contact.[6] Despite working as an outpatient nurse, it is less likely that she contracted COVID-19 at the workplace as symptom onset was three weeks after her last known patient contact. The incubation period of COVID-19 is estimated to be between 2 and 14 days. [7,8,9] Alternatively, she may have been exposed during self-imposed home isolation in Wuhan, however her apparent contacts including her husband and grandson were asymptomatic. Her grandson later tested negative for COVID-19. There is a likelihood that she was infected during transit to the quarantine facility at either the Wuhan International Airport or on her flight to the United States. Exposure at the quarantine facility seems less likely as that would reflect an incubation period of only a day.

Similar to the first documented cohort of COVID-19 patients linked to the Huanan market, our patient had leukopenia and thrombocytopenia, both of which recovered during the course of illness.[2] She notably developed lymphopenia on hospital day 6 , which also recovered. The rise in liver enzymes was more likely related to the disease course of COVID-19 than to drug-induced liver injury from remdesivir, as enzyme levels down trended to normal during daily antiviral infusions. No other clear implicating medications causing liver injury were utilized. Relative hypoxemia was noted by oxygen saturation, however, the patient never required supplemental oxygen. Chest imaging findings were also significant for 
infiltrates. In contrast to the reported cohort,[2] renal function in this patient remained normal during the disease course.

The clinical improvement in our patient coincided with the initiation of remdesivir on day 6 of hospitalization. However, it is unclear if this improvement can be attributed to the drug and/or to the natural course of COVID-19 infection. Ongoing investigational trials will help establish the efficacy of remdesivir and other antivirals in COVID-19 management. [10-12]

CDC-guided NP and OP samples of our patient from hospital day 1 onwards all tested negative for COVID-19 (Table 1). Although the patient was clinically ill enough to warrant continued hospitalization and initiation of the antiviral remdesivir; she would have met laboratory criteria to clear isolation precautions by hospital day 3 with two negative NP and OP specimens on two separate days. During hospital days 1 to 7, her high fevers and infectious signs in the setting of NP and OP negativity question whether the patient was infective at the time or whether these were manifestations of a prolonged, excessive cytokine and chemokine response that has been documented in other highly pathogenic coronaviruses including SARS-CoV and MERS-CoV.[13]

Patient's positive sputum RT-PCR for COVID-19 likely represented inactive viral shedding in the lower respiratory tract rather than active contagious virus given she had dramatic clinical improvement by hospital day 11. In 2003 SARS-CoV outbreak, viral shedding was detected in the respiratory, gastrointestinal, and urinary tracts for many weeks after onset of illness, while active transmission of infection was not noted after 2 weeks.[14] The pragmatic correlation of SARS-CoV-2 detection by RT-PCR in sputum, NP, and OP samples with infectivity is warranted to explore similar trends for COVID-19. Further research aimed at the understanding of transmission dynamics will help to guide screening practices and support clearance of infectivity.

\section{Conclusions}

Our experience with one of the earliest cases of COVID-19 in the United States offers insight into the pertinent clinical and laboratory findings of this novel disease entity. Use of remdesivir without any overt side effects in our patient, supports the ongoing clinical trials as a candidate therapeutic agent in COVID19. In addition, the case highlights possible areas for improvement in diagnosing coronavirus disease and robust protocols in establishing clearance of infection to facilitate removal of isolation precautions and safe return of patients to the community. These topics are pertinent for controlling disease spread and promoting public safety.

\section{Appendix}

* CDC guidelines for clinical specimen for diagnostic testing of COVID-19. For nasopharyngeal and oropharyngeal swabs, synthetic fiber swabs with plastic shafts were used and immediately placed into sterile tube containing 2-3 mL of viral transport media; each nasopharyngeal and oropharyngeal swab 
was kept in a separate vial. The specimens were refrigerated at $2-8^{\circ} \mathrm{C}$ and shipped overnight to the CDC on ice packs. For sputum samples, the patient expectorated directly into a sterile, leak-proof, screw-cap sputum collection cup, which was then refrigerated at $2-8^{\circ} \mathrm{C}$ and shipped overnight to the CDC. A RT-PCR assay, which was developed from publicly released virus sequence, was utilized in testing the clinical specimens.

\section{Abbreviations}

SARS-CoV-2: Severe Acute Respiratory Syndrome-related CoronaVirus 2

COVID-19: Coronavirus Disease 2019

WBC: White Blood Cell counts

ALC: Absolute Lymphocyte Counts

Tmax: Maximum temperature

NP: Nasopharyngeal

OP: Oropharyngeal

RT-PCR: Reverse Transcription Polymerase Chain Reaction

CDC : US Centers for Disease Control and Prevention

ANC: Absolute Neutrophil Count

AST : Aspartate Aminotransferase

ALT : Alanine Aminotransferase

SARS-CoV: Severe Acute Respiratory Syndrome CoronaVirus

MERS-CoV: Middle East Respiratory Syndrome CoronaVirus

\section{Declarations}

Conflict of Interest: We have no conflict of interest for publication of this manuscript

Funding: None

Patient Consent: Written informed consent was obtained from the patient for publication of this case report and any accompanying images. A copy of the written consent is available for review by the Editor in-Chief of this journal. 
Availability of data and materials: Not applicable

\section{Author Contributions:}

SS: acquisition and interpretation of data, literature search, and first draft. DB: critical revision FT: critical revision KK: acquisition of data, critical revision DA: critical revision RT: critical revision VG: interpretation of data, literature search, critical revision. All the authors were involved in manuscript preparation, have read and approved the final manuscript.

Acknowledgements: None

\section{References}

1. Chan JF, Yuan S, Kok KH, To KK, Chu H, Yang J et. al. A familial cluster of pneumonia associated with the 2019 novel coronavirus indicating person-to-person transmission: a study of a family cluster. Lancet. 2020; 395(10223): 514-523.

2. Huang C, Wang Y, Li X, Ren L, Zhao J, Hu Y et. al. Clinical features of patients infected with 2019 novel coronavirus in Wuhan, China. Lancet. 2020; 395(10223): 497-506.

3. Zhu N, Zhang D, Wang W, Li X, Yang B, Song J et. al. China Novel Coronavirus Investigating and Research Team. A Novel Coronavirus from Patients with Pneumonia in China, 2019. N Engl J Med. 2020; 382(8): 727-733.

4. World Health Organization. Coronavirus disease (COVID-2019) situation reports. https://www.who.int/docs/default-source/coronaviruse/situation-reports/20200331-sitrep-71-covid19.pdf?sfvrsn=4360e92b_4 (Accessed March 31, 2020)

5. Wang M, Cao R, Zhang L, Yang X, Liu J, Xu M et. al. Remdesivir and chloroquine effectively inhibit the recently emerged novel coronavirus (2019-nCoV) in vitro. Cell Res. 2020; 30(3): 269-271.

6. Lai C-C, Shih T-P, Ko W-C, Tang H-J, Hsueh P-R. Severe acute respiratory syndrome coronavirus 2 (SARS-CoV-2) and coronavirus disease-2019 (COVID-19): The epidemic and the challenges. Int J of Antimicrobial Agents. 2020. 105924.

7. Centers for Disease Control and Prevention. Coronavirus Disease 2019 (COVID-19) Situation Summary. (https://www.cdc.gov/coronavirus/2019-nCoV/summary.html) (accessed March 20, 2020)

8. Linton NM, Kobayashi T, Yang Y, Hayashi K, Akhmetzhanov AR, Jung SM et. al. Incubation Period and Other Epidemiological Characteristics of 2019 Novel Coronavirus Infections with Right Truncation: A Statistical Analysis of Publicly Available Case Data. J Clin Med. 202017 ; 9(2).

9. Lauer SA, Grantz KH, Bi Q, Jones FK, Zheng Q, Meredith HR et. al. The Incubation Period of Coronavirus Disease 2019 (COVID-19) From Publicly Reported Confirmed Cases: Estimation and Application. Ann Intern Med. 2020 Mar 10. doi: 10.7326/M20-0504. 
10. Study to Evaluate the Safety and Antiviral Activity of Remdesivir (GS-5734 $4^{\mathrm{TM}}$ ) in Participants with Moderate Coronavirus Disease (COVID-19) Compared to Standard of Care Treatment. (https://clinicaltrials.gov/ct2/show/NCT04292730) (accessed March 15, 2020)

11. Study to Evaluate the Safety and Antiviral Activity of Remdesivir (GS-5734 ${ }^{\mathrm{TM}}$ ) in Participants With Severe Coronavirus Disease (COVID-19). https://clinicaltrials.gov/ct2/show/NCT04292899? cond=remdesivir\&draw=2\&rank $=4$ (accessed March 15, 2020)

12. Lopinavir/ Ritonavir, Ribavirin and IFN-beta Combination for $\mathrm{nCoV}$ Treatment. https://clinicaltrials.gov/ct2/show/NCT04276688?cond=lopinavir\&draw=2\&rank=5 (accessed March $15,2020)$

13. Channappanavar R, Perlman S. Pathogenic human coronavirus infections: causes and consequences of cytokine storm and immunopathology. Semin Immunopathol. 2017; 39(5): 529539.

14. Chan KH, Poon LL, Cheng VC, Guan Y, Hung IF, Kong J et al. Detection of SARS coronavirus in patients with suspected SARS. Emerg Infect Dis. 2004; 10(2): 294-9.

\section{Tables}

Table 1: see figure 4

\section{Figures}

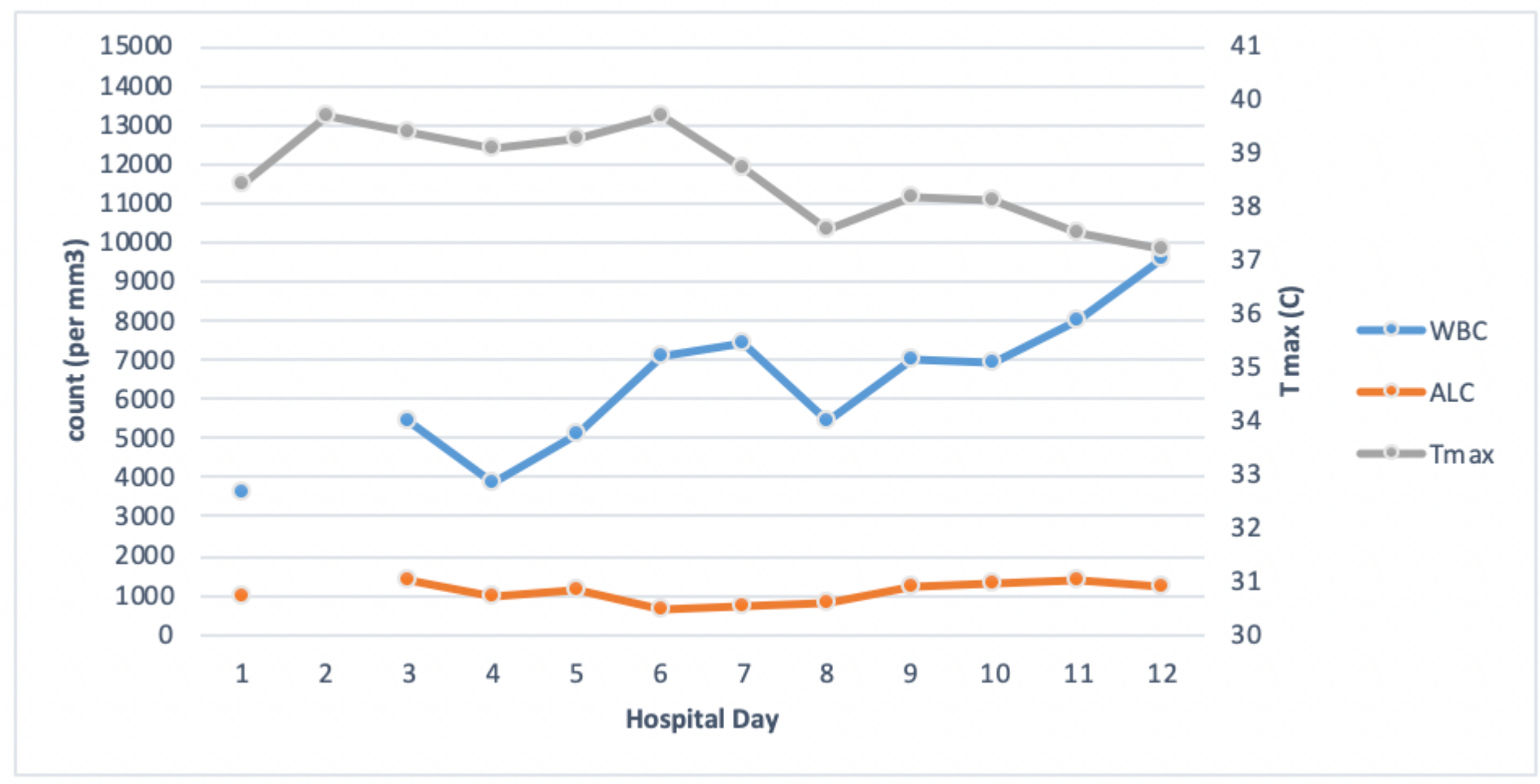


Maximum temperature (Tmax), white blood cell count (WBC) and absolute lymphocyte count (ALC) trend during hospitalization.

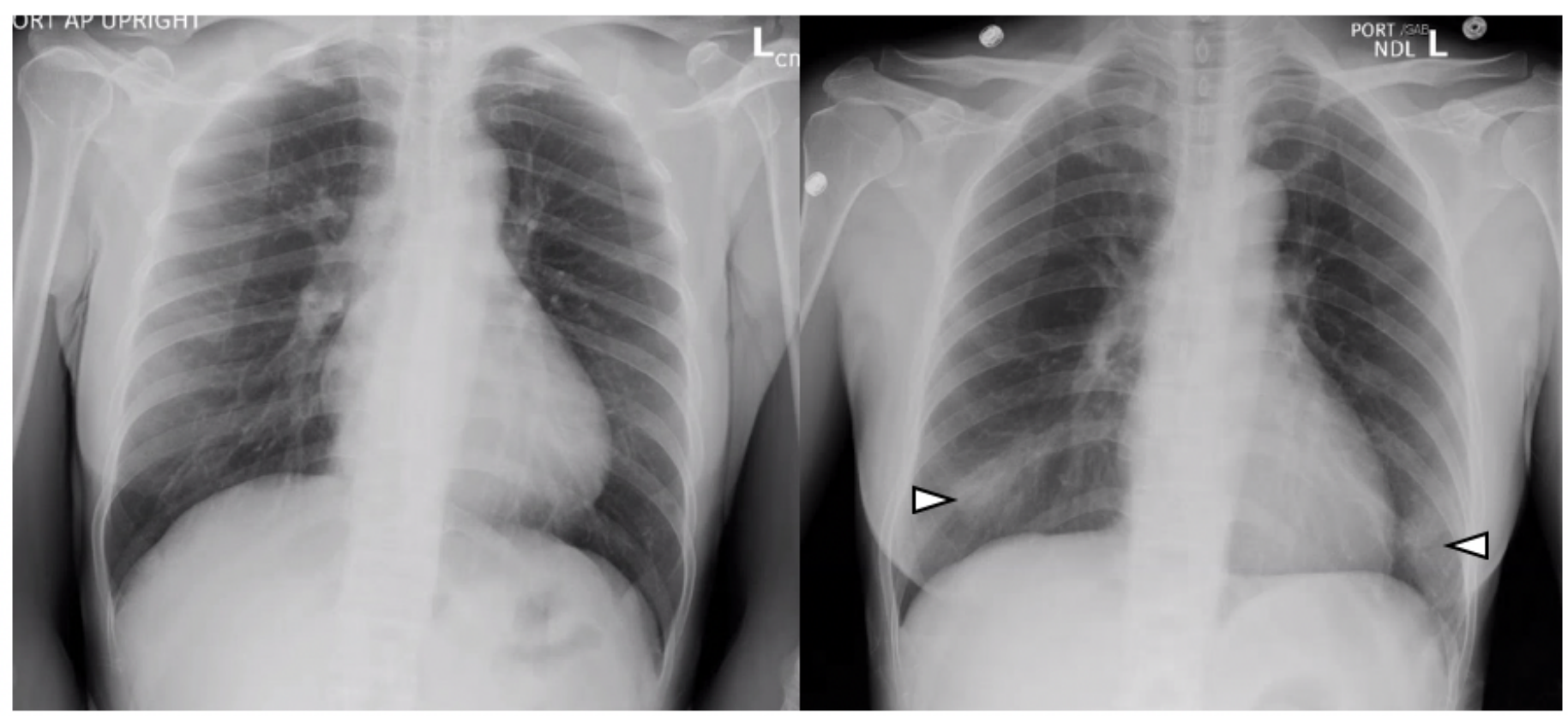

\section{A.}

B.

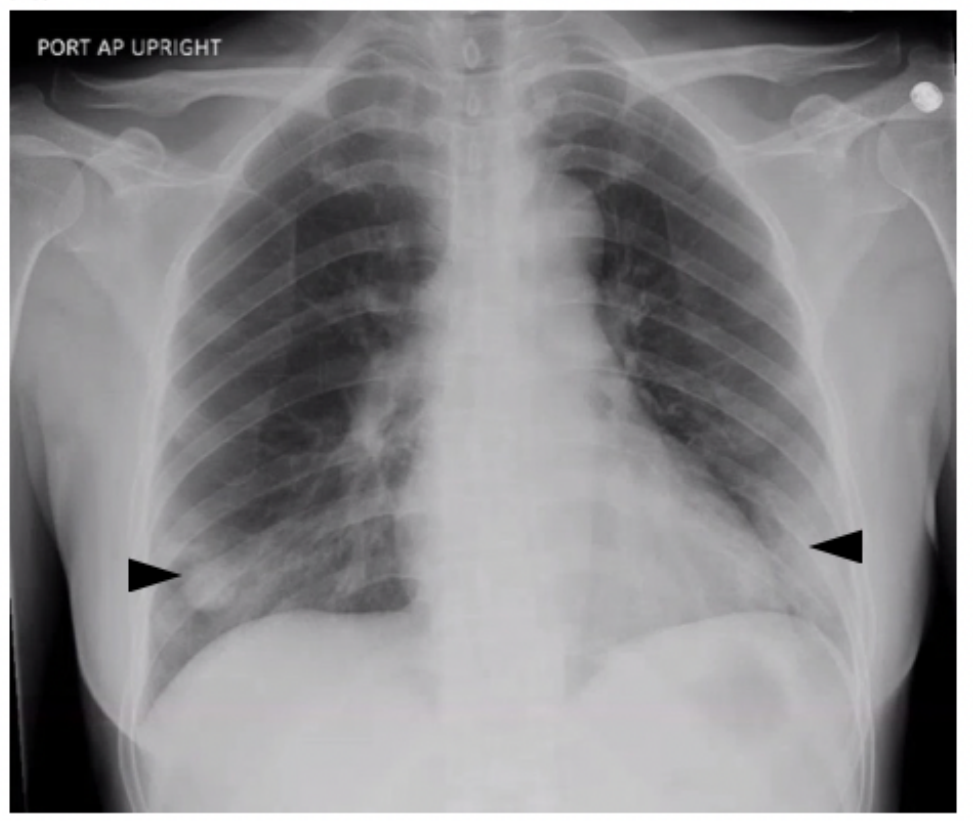

C.

Figure 2

Chest $\mathrm{X}$ ray from (A) Hospital day 1, (B) day 3, and (C) day 6 showing serial worsening bibasilar infiltrates. White arrows $=$ Developing infiltrates. Black arrows $=$ Worsening infiltrates . 


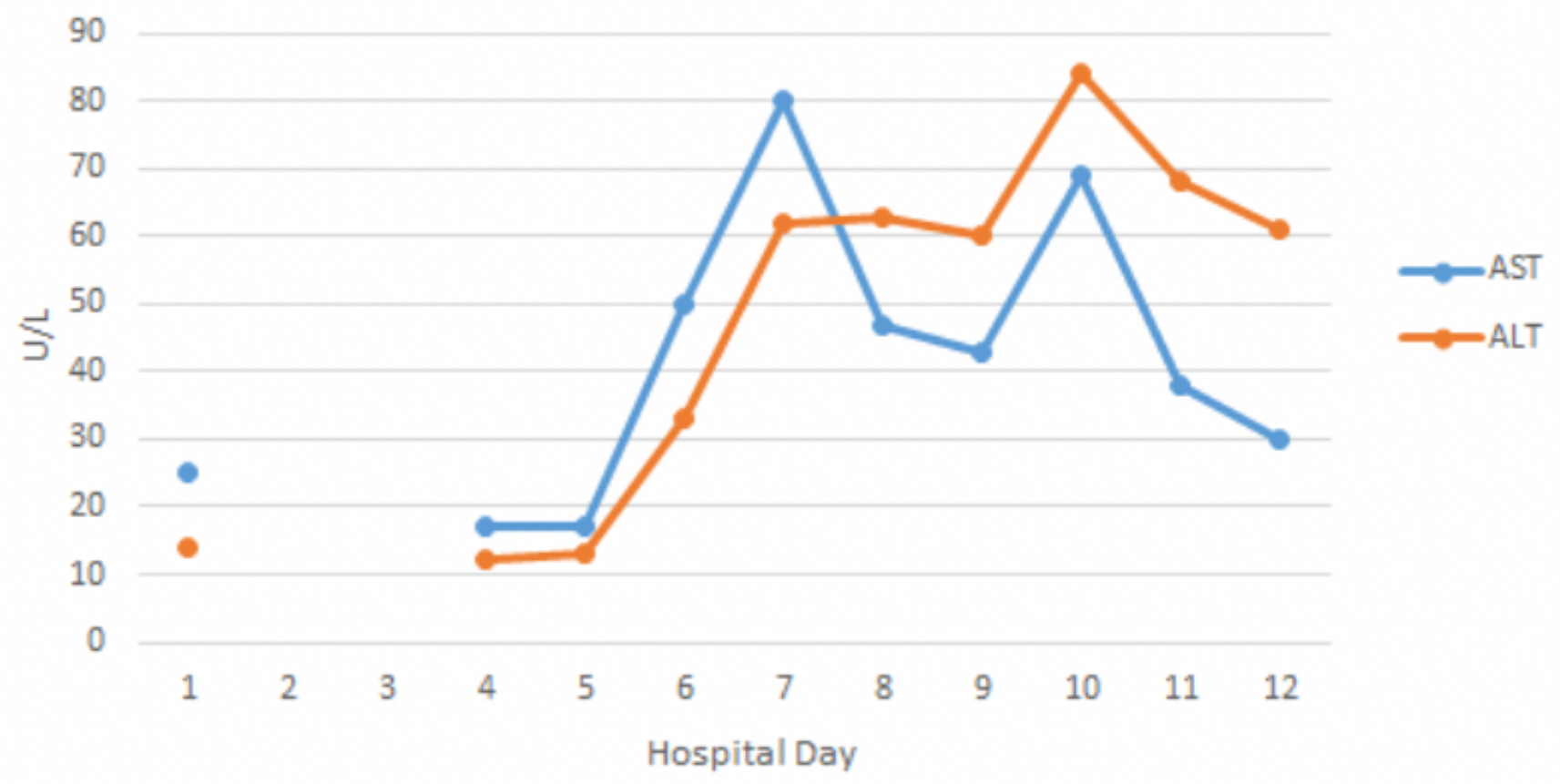

Figure 3

Liver function test (AST and ALT) trend during hospitalization.

\begin{tabular}{|l|l|l|l|l|l|l|l|l|}
\hline Test & $\begin{array}{l}\text { Day } \\
0^{* *}\end{array}$ & Day 1 & Day 3 & Day 5 & Day 7 & Day 8 & Day 10 & Day 11 \\
\hline NP Swab* & Pos & Neg & Neg & Neg & Neg & Neg & & \\
\hline OP Swab* & Pos & Neg & Neg & Neg & Neg & Neg & & \\
\hline Sputum* & & & & & & & Pos & Pos \\
\hline
\end{tabular}

Figure 4

(also called Table 1) Infection surveillance tests during hospitalization. Pos: Positive, Neg: Negative. * See appendix ${ }^{*}$ Day 0 correlates with day of illness onset (tests done at quarantine facility), Day 1 is initial day of hospitalization. 